\title{
Research Paper \\ Comparing the Effect of Exposure and Response Prevention and Paradoxical Time Table Therapy Techniques on the Obsessive-Compulsive Disorder Patients
}

\author{
Sadighe Ahmadi ${ }^{1}{ }^{\circledR}$, *Azarmidokht Rezaei ${ }^{1}$, Siamak Samani ${ }^{2}$, Sultanali Kazemi ${ }^{1}$
}

1. Department of Psychology, Marvdasht Branch, Islamic Azad University, Marvdasht, Iran.

2. Department of Educational Sciences, Shiraz Branch, Islamic Azad University, Shiraz, Iran.

\begin{tabular}{|l|l|l}
\hline $\begin{array}{c}\text { Use your devic to scan } \\
\text { and read thearticle online }\end{array}$ \\
Time Table Therapy Techniques on the Obsessive-Compulsive Disorder Patients (Persian)]. Quarterly of "The Horizon of Medical \\
Sciences". 2020; 26(3):244-259. https://doi.org/10.32598/hms.26.3.3093.3 \\
dof https://doi.org/10.32598/hms.26.3.3093.3
\end{tabular}

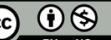

Received: 06 Aug 2019

Accepted: 07 Apr 2020

Available Online: $01 \mathrm{Jul} 2020$

Key words:

Counter-response technique, Exposure and Response Prevention (ERP), Paradoxical Time Table Technique, Obsessive-Compulsive Disorder (OCD)

\section{A B S TRACT}

Aims Obsessive-Compulsive Disorder (OCD) is one of the most common psychiatric disorders. Almost about five million Americans are identified with OCD every year. The purpose of this study was to compare the effectiveness of the Exposure and Response Prevention (ERP) and paradoxical time table therapy technique on OCD patients.

Methods \& Materials This experimental study was an extension of the multi-group pre-test post-test design. The statistical population of the study consisted of all OCD patients referred to private clinics and psychotherapy centers in Tehran in 2018, of whom 45 cases were selected through available sampling randomly assigned to three groups of 15. Patients in the first group received ERP ( 8 sessions) and patients in the second group received a paradoxical time table technique (4 sessions) individually. In the present study, the Maudsley Obsessive-Compulsive Inventory (1997) was used. The data were analyzed using Analysis of Covariance (ANCOVA) using SPSS software.

Findings The results showed the significant effectiveness of ERP and the paradoxical time table technique on the reduction of OCD $(P<0.001)$.

Conclusion Paradoxical time table therapy, as an alternative treatment of response prevention technique, can eliminate maladaptive thinking strategies about anxiety as well as uncompromising threat monitoring in OCD patients.

\section{Extended Abstract}

\section{Introduction}

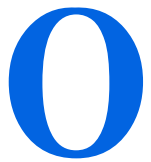

bsessive-Compulsive Disorder (OCD) is one of the most common psychiatric disorders, affecting nearly five million Americans each year [1]. Among all anxiety disorders, OCD occurs with the highest prevalence and rate of
1.5 and $3 \%$ of the adult population [3]. In a study of 1557 women aged 20-40 years in Ardabil city, the prevalence of OCD was reported to be 37.9 [4].

In a study on the prevalence of OCD in 692 students of the universities of Khoy city, 34.2\% had OCD [5]. In the study on the prevalence of OCD symptoms in the clinical population, the highest frequency was reported in the onset of OCD in the age group of 21-30 years [6]. Obsesreferral to medical centers [2]. OCD accounts for between

\section{* Corresponding Author:}

\section{Azarmidokht Rezaei, PhD.}

Address: Department of Psychology, Marvdasht Branch, Islamic Azad University, Marvdasht, Iran.

Tel: +98 (71) 43112201

E-mail: rezaee.azar@yahoo.com 
sions include a wide range of topics, such as pollution, illness, injury, moral issues, etc. [7].

According to the Diagnostic and Statistical Manual of Mental Disorders (DSM-6), OCD causes considerable suffering and significant disruption in normal life [8]. Due to the diversity of the clinical profile of this disease, several methods of treatment have been proposed, including psychological and pharmacological interventions and/or their combination [9], of which Exposure and Response Prevention (ERP) is of great importance. Behavioral therapies, ERP have consistently led to significant improvements in both obsessional thoughts and obsessional behavior $[10,11]$.

ERP was first performed by Meyer et al. on patients with an obsession with pollution and impurity and purity [12]. Foua et al. analyzed the results of 18 controlled studies and found that in $51 \%$ of patients, after the implementation of the ERP method, the symptoms of the disease disappeared or significantly improved [13]. The results of Laforst et al. study on three women with obsessional behavior confirmed the effectiveness of ERP on the samples [14].

Another method that can play a role in reducing the symptoms of OCD is the paradoxical time table technique. This technique is a new method for the treatment of OCD and little research has been done in this field [15]. It has been used as another method to treat OCD in this study, which introduced by Frankel and mainly refers to an intentionally obsessional behavior/response that is stressful and worrying [16]. Therefore, few studies have been conducted on the role of paradoxical time table technique in reducing OCD symptoms [17].

Paradoxical time table therapy is one of Frankel's "Searching for Meaning" concepts and it helps a person to understand that he or she is not like his or her perceived symptoms. In the paradoxical method, thoughts and feelings are typically designed to overshadow how one thinks about fear or unwanted behaviors [18]. "The moment we laugh at ourselves, some of the feelings of fear disappear," said Anne Gerber, and Frankel recommends that "the paradoxical intention must always be ironic" [19].

The results of Stifelman research showed that using a therapeutic approach with conflicting intentions can play a significant role in dealing with psychological problems [20].

Paradoxical therapy is used in a wide range of mental disorders, including sleep disorders [21], phobias [22], and especially OCD [23]. Given that OCD in all members of society reduces the efficiency of daily activities and disrupts normal life, the need for this research becomes clear. Although dif- ferent schools in today's clinical psychology claim to be effective, trying to differentiate between different schools and techniques effective in treating various psychological disorders seems to be a necessary task.

Few studies have compared the effectiveness of these two therapies on different factors and disorders. Most of these studies have examined them separately indicating the superiority of the ERP approach or paradoxical time table technique. Therefore, the present study aimed at comparing the effectiveness of the coping strategy using ERP and paradoxical time table technique on the obsessional behavior of people with OCD.

\section{Materials and Methods}

This research was a quasi-experimental study using the multi-group pre-test post-test design. The statistical population included all outpatients with OCD referred to private offices and clinics and psychotherapy centers of Tehran city in 2017-2018. Based on the cut-off point of scores (above 25) in the Maudsley Obsessive-Compulsive Inventory (MOCI) questionnaire, 45 of them were selected through available sampling and based on three indicators of test power (0.95), effect size (0.22), and the alpha error $(0.05)$ were entered into the study and divided randomly into three groups $(n=15$ per group): two experimental groups using ERP and paradoxical time table methods and one control group [24].

The inclusion criteria were as follows: the age of 20-40 years, agreement to cooperate in research, lack of other psychological disorders, and no use of psychiatric drugs. The exclusion criteria were the age of over 40 years or less than 20 years, the reluctance to participate in research, and using psychiatric drugs. This article was approved by the Islamic Azad University, Marvdasht Branch (Ethics Code: IR.IAU. MA.REC.1398.006).

To observe ethical considerations, the study participants were explained that they were participating in a study that sought to evaluate the effectiveness of the ERP and paradoxical time table therapy technique on obsessional behavior of people with OCD. They were also assured that all information would remain completely confidential. In addition, while answering the questions, they were assured that the research results would be published statistically and in general rather than individual conclusions, and also they are fully free to discontinue participating in the research at any stage they wish. The participants did not receive any other treatment during the sessions. After selecting the sample, the participants answered Maudsley Obsessive-Compulsive Inventory (MOCI), questionnaire (pre-test). Then, the two experimental groups were exposed to two types of interven- 
tions and finally, all three groups responded to the scale again and the results were compared. It is noteworthy at the end of the research, both ERP and paradoxical time table therapy techniques were provided for the control group.

\section{Research tools}

Maudsley Obsessive-Compulsive Inventory (MOCI): This questionnaire was developed by Hodgson and Radman to measure different types of obsessional-compulsive problems. This questionnaire consists of 30 items, half with the right key, and half with the wrong key. The score ranged from 0-30. In the initial validation at Maudsley Hospital, it was conducted on a sample of 50 neurotic patients and a sample of 50 nonclinical patients. In addition to an overall obsessive-compulsive score, the questionnaire provides four sub-scores for "reviewing, washing and cleaning, slownessrepetition, and skepticism". In a study on 40 patients, Hodgson and Radman found that the overall score of the questionnaire was sensitive to treatment changes.

Sanavio found a correlation of 0.70 , which was between the total scores on the Maudsley test and the Paddo test. The calculated reliability coefficient between the test and the retest was high (0.89). In Iran, Staky obtained the reliability of 0.85 for this tool by the re-test method. Dadfar also found the reliability coefficient of 0.84 for the whole test and the convergent validity of 0.87 with the Yale-Brown ObsessiveCompulsive Scale.

In the present study, Cronbach's alpha method was used to assess the reliability of the questionnaire, which was 0.74 for obsession, 0.62 for review, 0.62 for washing and cleaning, 0.70 for repetition, and 0.73 for skepticism.

\section{Exposure and Response Prevention (ERP) technique}

The ERP experimental group received eight 90-min sessions weekly and a treatment package was also provided. In this group, Ghahari's behavioral therapy techniques were used. The first session of the treatment included establishing a good relationship with clients, obtaining information about their history of OCD, explaining the etiology of OCD, teaching the logic, introducing the ERP technique, studying the level of motivation of the client to participate in the treatment, providing support resources, and signing the treatment contract. In the second session, a hierarchy of therapy was formed, in which a list of all the situations requiring confrontation was prepared based on their difficulty.

The patient ranked zero to 100 according to the level of anxiety expected in a situation. Based on the difficulty of ranking, the therapist and patient decided to address each situation in one of the treatment sessions. In the third to seventh sessions, the patient was confronted with anxious situations that were identified for each session. During each session, anxiety levels were measured every $10 \mathrm{~min}$, and when the anxiety level dropped to an acceptable level and the treatment atmosphere tended to progress, an anxious situation was presented. At the end of each session, the therapist and the patient agreed on the homework needed to be done before the next session, and at the beginning of each session, the homework was reviewed. In the eighth session, the therapists prepared clients for the completion of the course of treatment and suggested strategies to maintain treatment outcomes.

\section{Paradoxical time table technique}

The paradoxical time table experimental group received four 90-min sessions of paradoxical time table technique weekly and the treatment package based on the teachings of Mohammad Ali Besharat [25]. In this treatment, the patient, in addition to performing his/her obsessive behaviors, specified three times a day to do the same behavior at a certain time that was usually 3-30 min a day, which did not change until the end of treatment, and if the patient failed to be on time, he/she stopped practicing it, similar to what is advised for taking antibiotics. The important point is that the patient should experience and deal with his/her negative feelings (guilt, remorse, embarrassment, ridicule, etc.) during the procedure. The scheduling program (time table) allows involuntary obsessive-compulsive behaviors to be performed voluntarily; that is, voluntary behavior is available to the patient, who like many voluntary behaviors may prefer not to do them.

\section{Data analysis}

In the present study, descriptive statistics, including frequency, averages, etc. were used to classify information and inferential statistics were used to analyze the data and review the research questions and hypotheses. The data obtained from the pre-test, post-test stages were analyzed by Analysis of Covariance (ANCOVA).

Then, the Bonferroni post-hoc was used to compare the effectiveness of the techniques in the experimental groups. Also, the Kolmogorov-Smirnov test was used for checking the normal distribution of data, Levene's test for the homogeneity of variance of post-tests research variables, and the Cruitt-Bartlett test for testing the standard correlation between the dependent variables. It should be noted that all statistical calculations were performed using SPSS V. 18 software. 


\section{Results}

The demographic characteristics of the samples are provided in Table 1.

According to the results of Table 2, there was a significant difference between the pre-test and post-test scores $(\mathrm{P}=0.000)$ in the ERP and paradoxical time table therapy groups. There was a significant difference between the scores of washing and cleaning subscales in the pre-test than the post-test and follow-up stages $(\mathrm{P}=0.001)$. There was a significant difference between slowness-repetition scores in the pre-test than the post-test and follow-up $(\mathrm{P}=0.002)$. A significant difference was found between the pre-test scores of the doubt-accuracy subscale compared with the post-test and follow-up stages ( $\mathrm{P}=0.001)$. There was also a significant difference between obsession scores in the post-test than the follow-up stages $(\mathrm{P}=0.004)$. Moreover, there was a significant difference between the review scores in the two pre-test and follow-up stages in both experimental groups $(\mathrm{P}=0.002)$. A significant difference was observed between washing and cleaning $(\mathrm{P}=0.002)$ and slowness-repetition $(\mathrm{P}=0.04)$ in these two stages. There was no significant difference between the scores of doubt-accuracy in these two stages $(\mathrm{P}=0.06)$. We found also a significant difference between obsession scores in pre-test and follow-up stages $(\mathrm{P}=0.001)$.

The results of Table 3 showed that the difference between the mean scores of review between the ERP and paradoxical time table therapy groups $(\mathrm{P}=0.19)$ and the ERP and control groups $(\mathrm{P}=0.11)$ was not significant. The difference between the mean scores of washing and cleaning in the ERP and paradoxical time table therapy groups was not significant $(\mathrm{P}=0.79)$, whereas it was significant in the ERP and control groups $(\mathrm{P}=0.001)$. The difference between the mean scores of the paradoxical time table therapy and control groups was significant $(\mathrm{P}=0.001)$. Also, the ERP and paradoxical time table therapy groups $(\mathrm{P}=0.25)$ and ERP group and control groups $(\mathrm{P}=0.001)$ showed a significant difference in the mean scores of slowness-repetition.

The difference between the mean scores of doubt-accuracy in the paradoxical time table therapy and control groups $(\mathrm{P}=0.001)$ and the ERP and paradoxical time table therapy groups $(\mathrm{P}=0.009)$ was significantly different.

The difference between the mean scores of obsessions in the ERP and paradoxical time table therapy groups $(\mathrm{P}=0.004)$ and the ERP and control groups $(\mathrm{P}=0.001)$ was also significant.

\section{Discussion}

The aim of this study was to compare the effectiveness of the ERP technique and the paradoxical time table therapy on the obsessional behavior of people with OCD. The results of ANCOVA showed the significant effect of treatment between the three research groups. Also, the results of the fol-

Table 1. Demographic characteristics of the samples

\begin{tabular}{|c|c|c|c|c|}
\hline \multirow{3}{*}{ Variables } & \multirow{3}{*}{ Indicators } & \multicolumn{3}{|c|}{ Groups } \\
\hline & & \multicolumn{3}{|c|}{ No. (\%) } \\
\hline & & $\begin{array}{l}\text { Exposure and Response } \\
\text { Prevention (ERP) }\end{array}$ & $\begin{array}{l}\text { Paradoxical Time } \\
\text { Table Therapy }\end{array}$ & Control \\
\hline \multirow{3}{*}{ Education } & Diploma & $9(60)$ & $8(3.53)$ & $5(3.33)$ \\
\hline & Bachelor's degree & $4(7.26)$ & $4(7.26)$ & $8(3.53)$ \\
\hline & Higher than bachelor's degree & $2(3.13)$ & $3(20)$ & $2(3.13)$ \\
\hline \multirow{2}{*}{ Gender } & Female & $11(3.73)$ & $11(3.73)$ & $10(7.66)$ \\
\hline & Male & $4(7.26)$ & $4(7.26)$ & $5(3.33)$ \\
\hline \multirow{3}{*}{ Occupation } & Housewife & $8(3.53)$ & $8(3.53)$ & $8(3.53)$ \\
\hline & Unemployed & $2(3.13)$ & $2(3.13)$ & $1(7.6)$ \\
\hline & Employed & $5(3.33)$ & $5(3.33)$ & $6(40)$ \\
\hline
\end{tabular}


Table 2. Mean and standard deviation of the Maudsley Obsessive-Compulsive Inventory scores ( $\mathrm{n}=15$ per group)

\begin{tabular}{|c|c|c|c|c|}
\hline \multirow{2}{*}{ Subscales } & \multirow{2}{*}{ Groups } & \multicolumn{3}{|c|}{ Mean $\pm S D$} \\
\hline & & Pre-test & Post-test & Follow-Up \\
\hline \multirow{3}{*}{ Review } & ERP & $5.4 \pm 1.45$ & $4.4 \pm 1.24$ & $4.33 \pm 1.11$ \\
\hline & Paradoxical Time Table Therapy & $5.2 \pm 1.08$ & $3.7 \pm 1.16$ & $4.2 \pm 1.09$ \\
\hline & Control & $5.33 \pm 1.04$ & $4.93 \pm 1.27$ & $5.86 \pm 0.99$ \\
\hline \multirow{3}{*}{ Washing and cleaning } & ERP & $6.46 \pm 1.99$ & $4.86 \pm 1.54$ & $5.46 \pm 1.05$ \\
\hline & Paradoxical Time Table Therapy & $6.4 \pm 1.92$ & $4.6 \pm 1.50$ & $5.26 \pm 1.66$ \\
\hline & Control & $6.2 \pm 1.16$ & $6.33 \pm 1.92$ & $7.06 \pm 1.30$ \\
\hline \multirow{3}{*}{ Slowness - Repetition } & ERP & $3.93 \pm 1.22$ & $3.06 \pm 1.30$ & $3.13 \pm 1.21$ \\
\hline & Paradoxical Time Table Therapy & $4.33 \pm 0.27$ & $2.66 \pm 1.40$ & $3.00 \pm 0.48$ \\
\hline & Control & $5.06 \pm 2.52$ & $4.26 \pm 0.88$ & $3.93 \pm 1.90$ \\
\hline \multirow{3}{*}{ Doubt - accuracy } & ERP & $4.13 \pm 1.21$ & $3.33 \pm 1.11$ & $3.26 \pm 1.72$ \\
\hline & Paradoxical Time Table Therapy & $4.00 \pm 1.37$ & $2.46 \pm 1.21$ & $2.93 \pm 0.88$ \\
\hline & Control & $4.06 \pm 1.90$ & $4.86 \pm 1.95$ & $3.73 \pm 1.72$ \\
\hline \multirow{3}{*}{ Obsession } & ERP & $17.73 \pm 3.10$ & $13.53 \pm 2.53$ & $14.26 \pm 2.17$ \\
\hline & Paradoxical Time Table Therapy & $17.53 \pm 1.99$ & $12.00 \pm 1.18$ & $13.4 \pm 1.59$ \\
\hline & Control & $17.86 \pm 2.14$ & $18.13 \pm 2.14$ & $18.26 \pm 2.94$ \\
\hline
\end{tabular}

ERP: Exposure and Response Prevention

low-up stage showed that the paradoxical time table therapy was more effective than the ERP technique (Table 4).

Regarding the effect of ERP on preventing obsessional behavior in people with OCD, the findings of this research were in line with the findings of Tice [10] and Blackey et al. [11]. Also, considering the effectiveness of the paradoxical time table therapy on the obsessional behavior of these people, our findings were consistent with the findings of Abramowitz et al. [15] and Fisher and Wells [17].

To explain this finding, it can be said that behavioral pattern is a method in explaining and treating the OCD. According to this pattern, obsessional thoughts are conditional stimuli. A relatively neutral stimulus is associated with fear and anxiety through the process of respondent conditioning and event pairing that is naturally harmful and anxious. Thus, previously neutral thoughts and objects become conditioned stimuli and are able to arouse anxiety and discomfort. However, obsessional behavior occurs differently; the person realizes that a particular action reduces the anxiety associated with an obsession. The anxiety relief, which acts as a secretive driver, strengthens the OCD. Gradually, this practice becomes a
Quarterly of

The Horizon of Medical Sciences consistent learned pattern of behavior due to its usefulness in reducing a painful secondary stimulus (anxiety).

In the present study, in explaining the greater effectiveness of the paradoxical time table therapy than the ERP technique, it should be said that in this approach, instead of challenging the treatment with disturbing thoughts and dysfunctional beliefs, it deals with thoughts to prevent resistance or complex perceptual analysis to eliminate inconsistent thinking strategies about anxiety as well as inflexible monitoring of the threats.

It should be noted that negative beliefs cannot clearly explain the pattern of thinking and subsequent responses. The factors that control thinking and change the state of mind should also be considered, which is the most important approach of the paradoxical time table therapy.

One of the reasons that the ERP technique was more effective in reducing obsessional behavior in people with OCD is the use of exposure technique for OCD patients or those with anxiety disorders. It provides an opportunity for their 
Table 3. The results of the ANCOVAfor the OCD and its subscales in the post-test and follow-up

\begin{tabular}{|c|c|c|c|c|c|c|}
\hline $\begin{array}{l}\text { Measurement } \\
\text { Time }\end{array}$ & Subscales & $\begin{array}{l}\text { Total Square } \\
\text { Footage }\end{array}$ & $\begin{array}{l}\text { Sum of } \\
\text { Squares }\end{array}$ & $\begin{array}{c}\text { Total square } \\
\text { Footage }\end{array}$ & $\mathbf{F}$ & Level of Significance \\
\hline \multirow{5}{*}{ Post-test } & Review & 11.00 & 2 & 5.5 & 4.42 & 0.01 \\
\hline & Washing and cleaning & 29.57 & 2 & 14.78 & 11.4 & 0.001 \\
\hline & Slowness - Repetition & 15.44 & 2 & 7.72 & 8.36 & 0.001 \\
\hline & Doubt - accuracy & 27.79 & 2 & 13.89 & 12.07 & 0.001 \\
\hline & Obsession & 222.34 & 2 & 111.17 & 52.15 & 0.001 \\
\hline \multirow{5}{*}{ Follow-up } & Review & 16.68 & 2 & 8.34 & 7.70 & 0.002 \\
\hline & Washing and cleaning & 24.29 & 2 & 12.14 & 7.49 & 0.002 \\
\hline & Slowness - Repetition & 7.53 & 2 & 3.76 & 3.5 & 0.04 \\
\hline & Doubt - accuracy & 6.00 & 2 & 3.00 & 2.96 & 0.06 \\
\hline & Obsession & 157.73 & 2 & 78.26 & 21.21 & 0.001 \\
\hline
\end{tabular}

Table 4. Follow-up test results

\begin{tabular}{|c|c|c|c|c|}
\hline Dependent Variables & Group I & Group J & Mean Difference & Significance Level \\
\hline \multirow{4}{*}{ Review } & & 3 & 0.54 & 0.19 \\
\hline & 2 & & & \\
\hline & & 1 & -0.7 & 0.11 \\
\hline & 3 & 1 & -1.52 & 0.005 \\
\hline \multirow{4}{*}{ Washing and cleaning } & & 3 & 0.1 & 0.79 \\
\hline & 2 & & & \\
\hline & & 1 & -1.77 & 0.001 \\
\hline & 3 & 1 & -1.88 & 0.001 \\
\hline \multirow{4}{*}{ Slowness - repetition } & & 3 & 0.41 & 0.25 \\
\hline & 2 & & & \\
\hline & & 1 & -1.04 & 0.001 \\
\hline & 3 & 1 & -1.46 & 0.001 \\
\hline \multirow{4}{*}{ Doubt - accuracy } & & 3 & 1.08 & 0.009 \\
\hline & 2 & & & 0001 \\
\hline & & 1 & -0.88 & 0.001 \\
\hline & 3 & 1 & -1.97 & 0.001 \\
\hline \multirow{4}{*}{ Obsession } & & 3 & 1.76 & 0.004 \\
\hline & 2 & & & \\
\hline & & 1 & -3.89 & 0.001 \\
\hline & 3 & 1 & -5.56 & 0.001 \\
\hline
\end{tabular}


minds to analyze the various dimensions of anxious stimuli and consequently provides an appropriate response.

Obsessional thoughts and behaviors are very real and natural for the patient; however, they are annoying and pathogenesis. Also, obsessional thoughts and behaviors are not commanding, that is, in real life, no one instructs an obsessive person to wash his hands ten times, check the windows, or take a shower five times a day.

When the therapist uses the paradoxical time table therapy technique and tells the patient to do the same obsessive things, but in pre-determined times, his/her actions and thoughts are done according to the therapist's instructions, not based on the obsessive attack. For this reason, the ERP is significantly effective in reducing the obsessional behavior in people with OCD [26].

Batiani and Guttman [26] in a review of 37 studies found that 25 of them reported paradoxical time table technique with useful clinical results and as the most effective psychotherapeutic methods.

Fabri [27] and Batiani and Guttman [26] stated that the paradoxical time table technique involves different types of psychotherapies, including cognitive-behavioral therapy. In this regard, Asher [23] showed that this technique is a very appropriate treatment for people with OCD and helps them to face and challenge their problems and ultimately modifies their inefficient beliefs. This treatment is strongly supported by the American Psychiatric Association (APA) [18].

\section{Conclusion}

The higher mean scores of the paradoxical time table group compared with the ERP and control groups in the post-test showed the effectiveness of this treatment approach on patients with OCD.

Given the stability of the mean scores in the control group members, it can be concluded that OCD, if left untreated, is a constant problem that over time leads to its stabilization and even its development. This emphasizes the fact that the possibility of recovery from OCD, without the intervention of therapeutic factors, is very low, at least in adulthood.

This research had some limitations. It was conducted in Tehran city and among the subjects with OCD; thus, the results should be generalized cautiously to other communities. Also, many of the dominant psychological factors remained uncontrolled. Another limitation is that the exact number of people in the community with OCD in Tehran city is not available and the lack of cooperation of some counseling centers and people with OCD also affected the research.

It is suggested that in the treatment of people with OCD, a paradoxical time table technique be applied so that the patients may benefit from the results of this research. Based on the results of this study, it is suggested to pay more attention to the use of paradoxical time table technique rather than ERP in reducing the symptoms of OCD in counseling centers.

Finally, this treatment can be considered as an adjunctive therapy that can be used to reduce the severity of symptoms obsessive along with other treatments in educational centers, such as schools, universities, and community health centers by psychologists, psychiatrists, and other therapists. It is suggested that in future studies, variables, such as the severity of obsession and emotional factors be considered as important factors in the perseverance of obsession.

\section{Ethical Considerations}

\section{Compliance with ethical guidelines}

This article was approved by the Islamic Azad University, Marvdasht Branch (Ethics Code: IR.IAU. MA.REC.1398.006).

\section{Funding}

This article was extracted from the $\mathrm{PhD}$. thesis of Sedigheh Ahmadi at the Department of Psychology, Marvdasht Branch, Islamic Azad University, Marvdasht.

\section{Authors' contributions}

Data collection, article writing, review of the final version: Sedigheh Ahmadi; Original idea, article writing, review of the final version: Azarmidokht Rezaei; Data interpretation, article writing, review of the final version: Siamak Samani; Study design, article writing, review of the final version: Sultan Ali Kazemi.

\section{Conflicts of interest}

The authors declared no conflict of interest. 


\title{
مقايسه اثربخشى تكنيك مواجها همراه جلوكيرى از هاسخ واسخ و تكنيك يار ادوكسيكال تايم تيبل بر

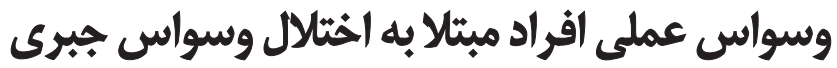

صديقه احمدى' (1)، •آذرميدخت رضايى'، سيامك سامانى'، سلطانعلى كاظمى'

ا. ا.تروه روانشناسى، واحد مرودشت، دانشعاه آزاد اسلامى، مرودشت، ايران.

ب. كُوره علوم تربيتى، واحد شيراز، دانشكاه آزاد اسلامى، شيرازا، ايران.

\begin{abstract}
حكبد

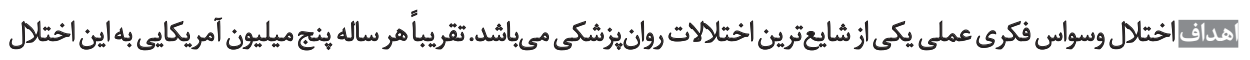

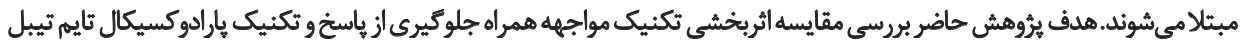

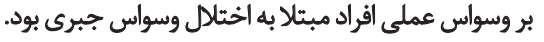

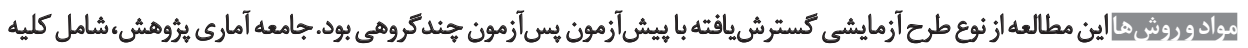

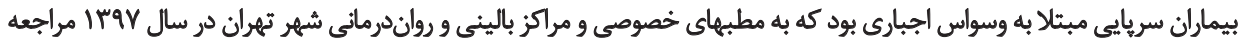

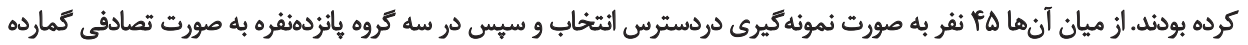

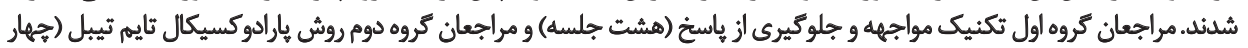

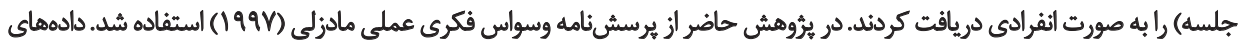

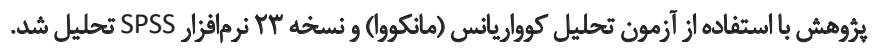

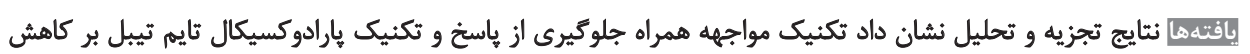

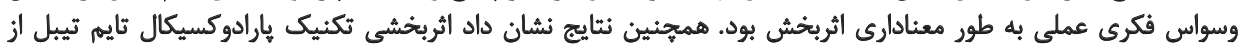

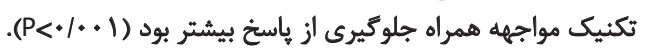

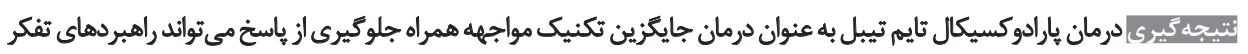

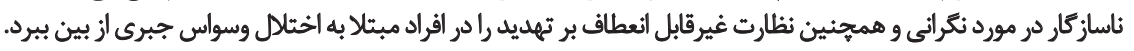

تاريخ دريافت: 10 مر داد

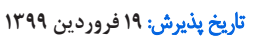

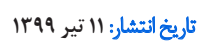

كليدوازوهها: مواجهه همراه جلوكيرى

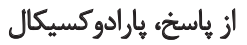

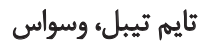

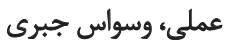

[ه]]. در بررسى ميزان شيوع علائم وسواس در جمعيت بالينى،

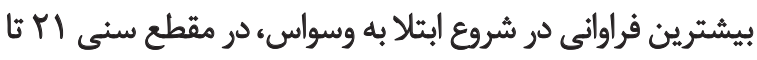

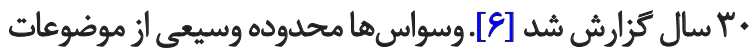

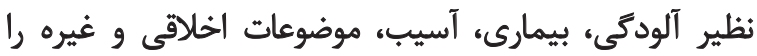

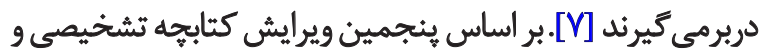

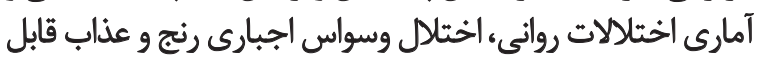

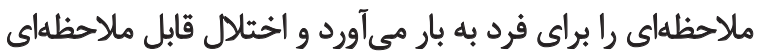

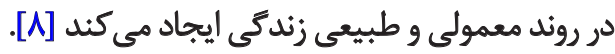
به دليل متنوع بودن نيمرخ بالينى اين بيمارى، شيوههاى

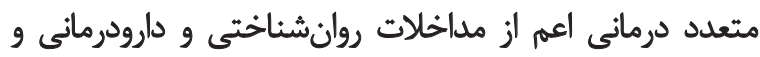

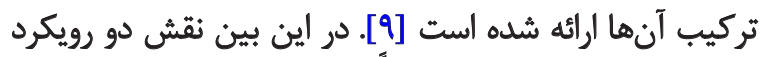

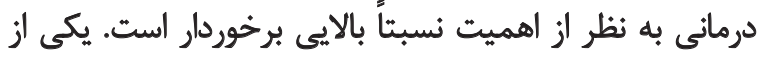

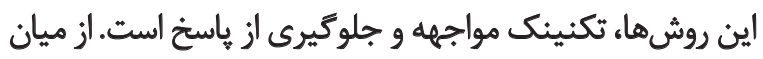

dales

اختلال وسواس فكرى عملى يكى از شايعترين اختلالات

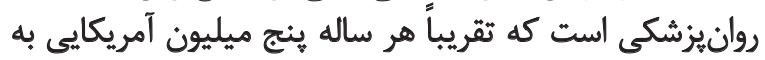

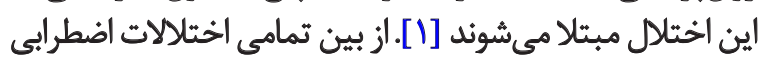

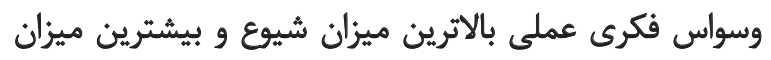

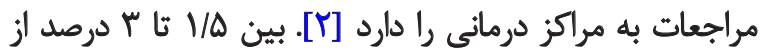

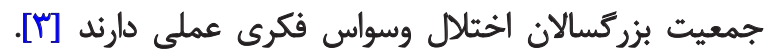

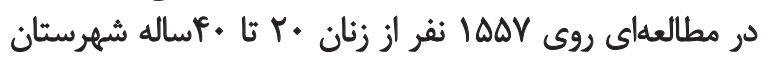

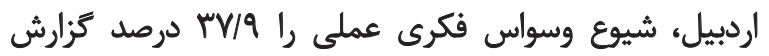

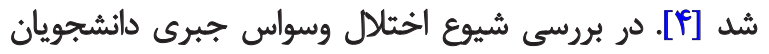

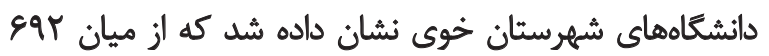

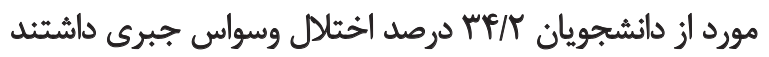

$$
\begin{aligned}
& \text { : نويسئده مسئول: }
\end{aligned}
$$

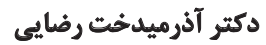

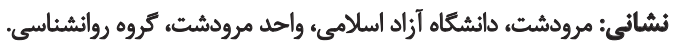

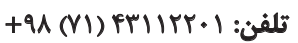

$$
\begin{aligned}
& \text { يست الكترونيكي: rezaee.azar@yahoo.com }
\end{aligned}
$$


متناقض در برخورد با مشكلات روانى، مي تواند نقش معنى داردارى

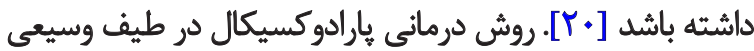

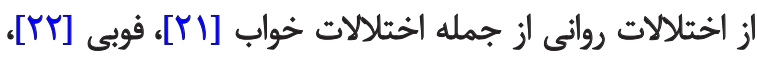

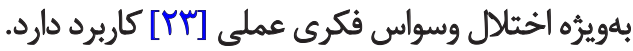
با توجه به آنكه اختلال وسواس اجبارى در تمام اقشار جامعه

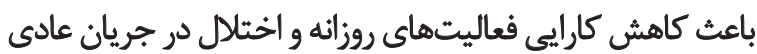

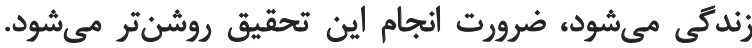

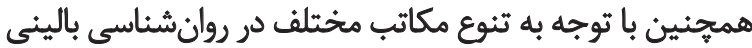

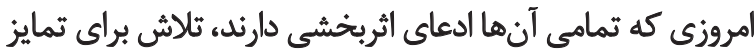

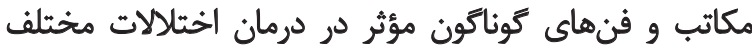

$$
\text { روانشناختى امرى ضرورى به نظر مى مئرسد. }
$$

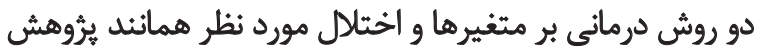

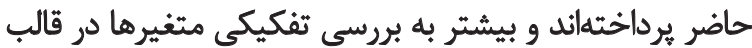

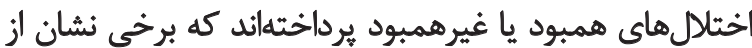

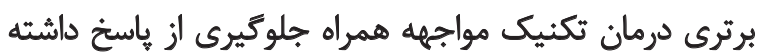

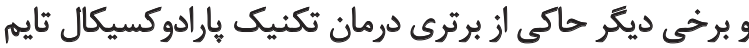

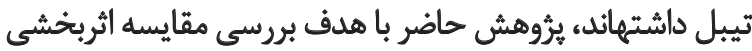

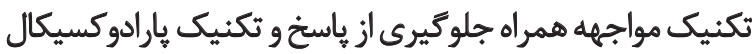

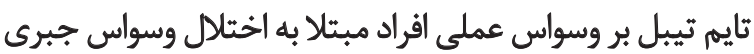

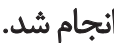

\section{مواد و روشها}

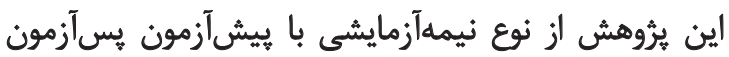

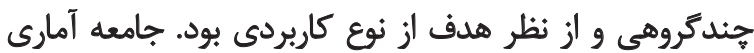

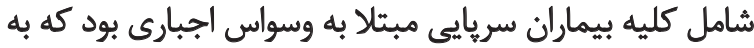

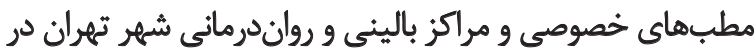

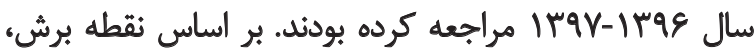

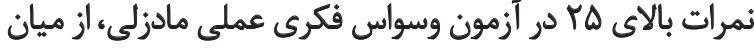

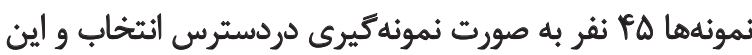

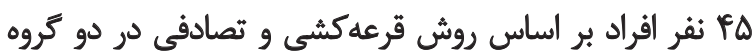

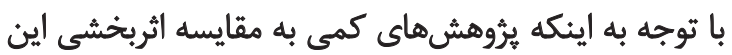

درمانهاى رفتارى، رويارويى و جلوكيرى از ياسخ به طور يُيوسته

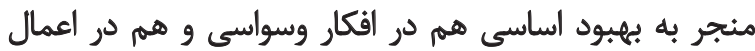

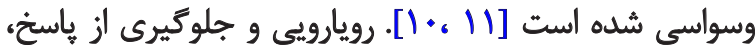

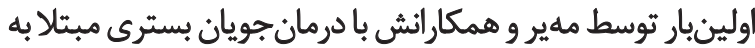

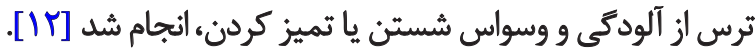

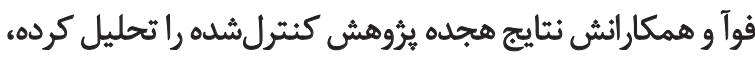

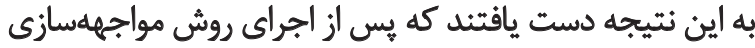

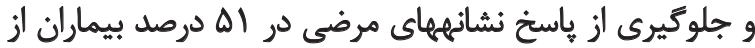

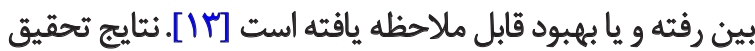

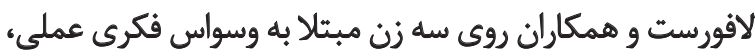

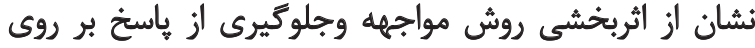

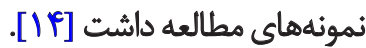

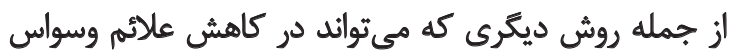

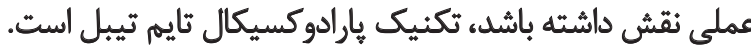

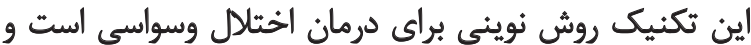

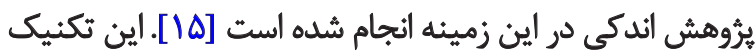

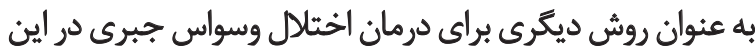

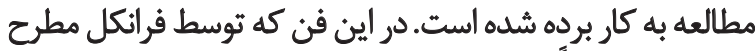

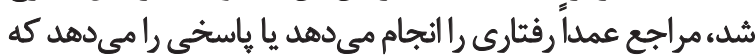

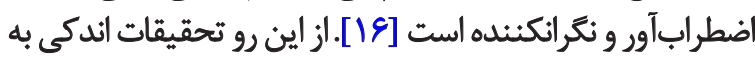

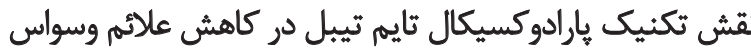

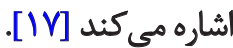

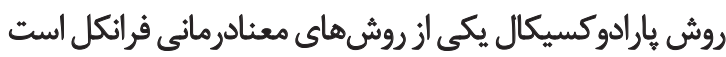

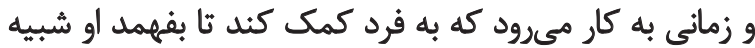

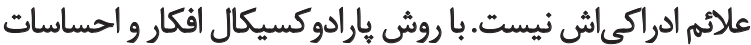

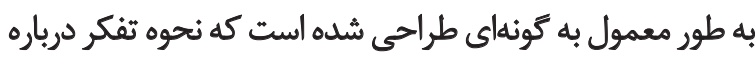

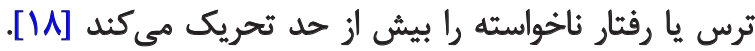

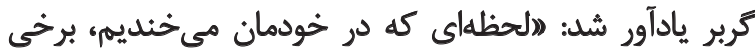

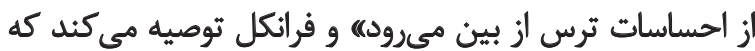

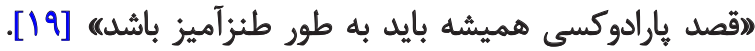
نتايج تحقيق استيفلمن نشان داد استفاده از رويكرد درمانى قصد بادي بادي

جدول ا. برونداد نرم|فزار G power جهت تعيين حجم نمونه

\begin{tabular}{|c|c|}
\hline اندازهها & مفروضهها \\
\hline . & Effect size f2(v) \\
\hline $.1 . \Delta$ & error prob $\alpha$ \\
\hline.$/ 9 \Delta$ & Power (1- $\beta$ error prob) \\
\hline r & Number of group \\
\hline r & Response variable \\
\hline PA & Total sample size \\
\hline
\end{tabular}




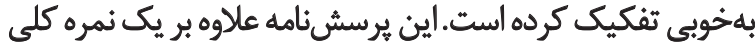

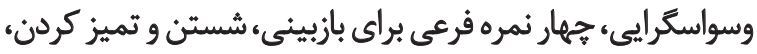

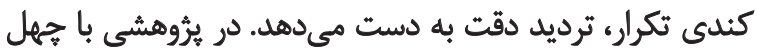

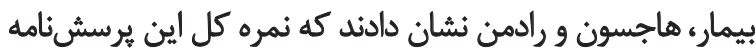

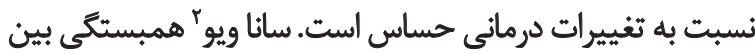

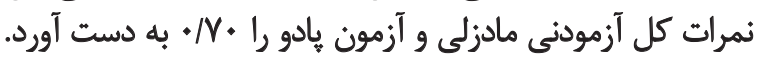

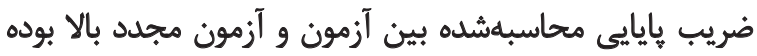

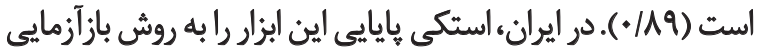

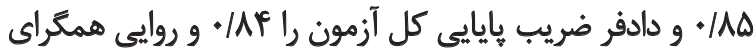

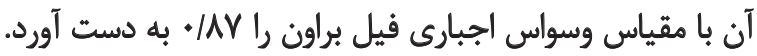

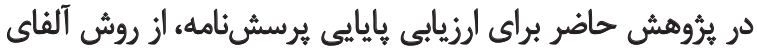

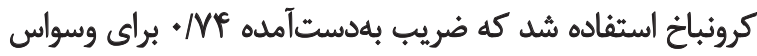

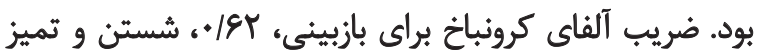

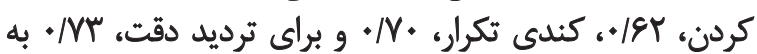
دست آمد.

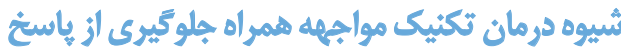

كروهاى آزمايشى مواجههاسازى و جلوكيرى از هاسخ، هشت

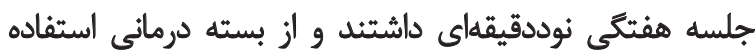
كردند. در اين كروه بر اساس فنون رفتئار درمانى قهارى انجام شد.

جلسه اول درمان شامل برقرارى رابطه خوب با مراجع و به

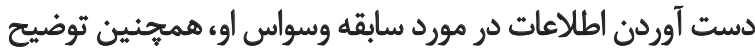

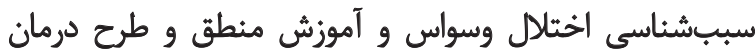

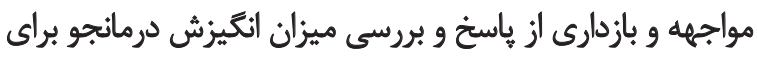

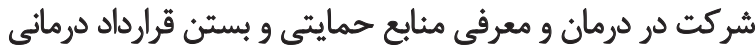

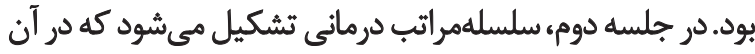

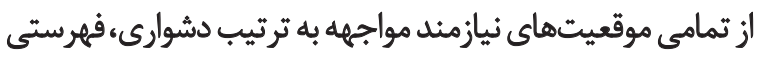

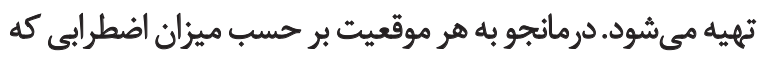

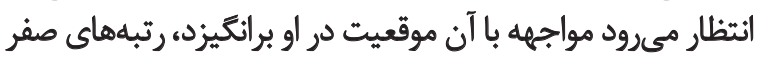

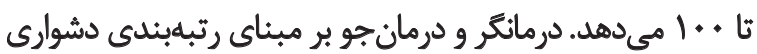

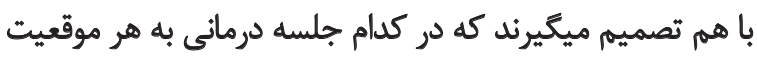

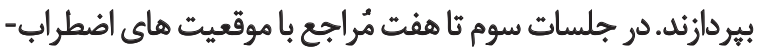

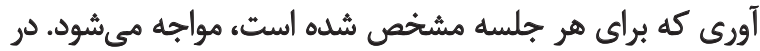

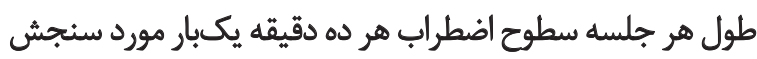

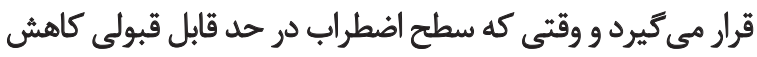

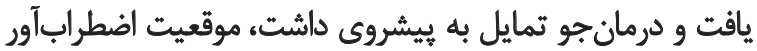

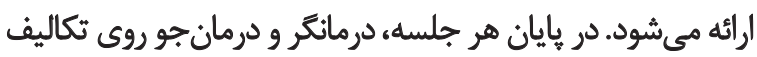

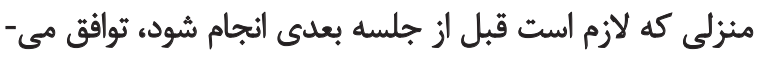

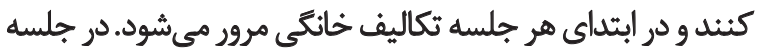

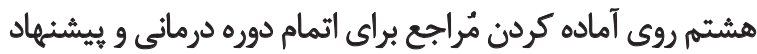

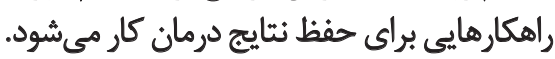

2. Sanavio
آزمايشى (كروه تكنيك مواجهه و جلوكيرى از ياسخ (يانزده نفر)

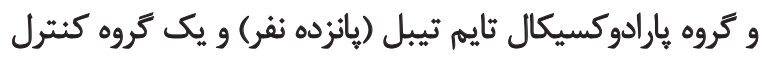

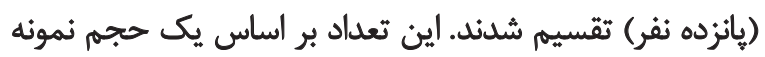

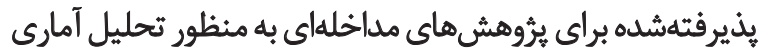

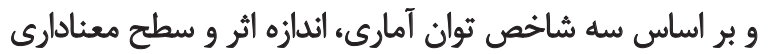

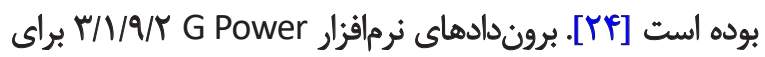
حداقل نمونه در جدول شماره اكزارش شرأ شده است.

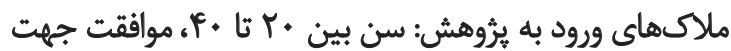

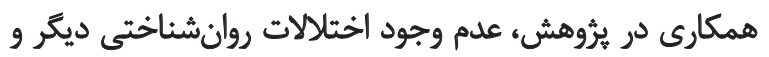

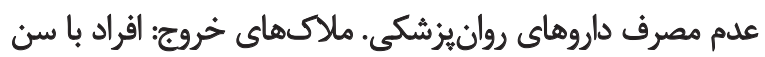

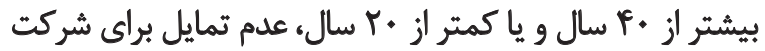

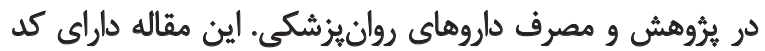

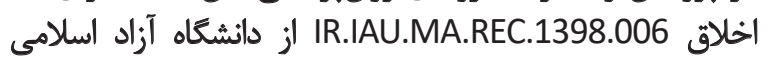

واحد مرودشت بود.

جهت رعايت ملاحظات اخلاقى به شركت كنيندكان بثروهش

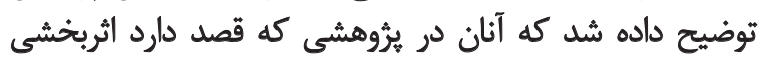

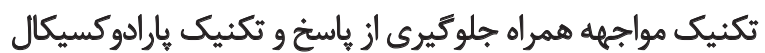

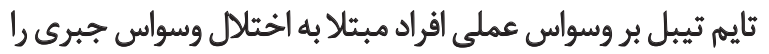

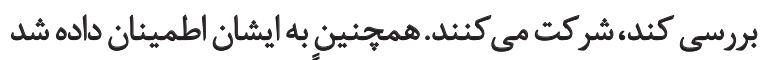

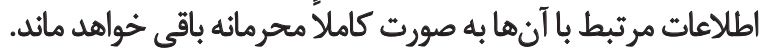

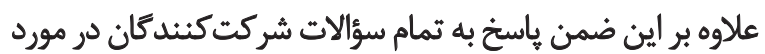

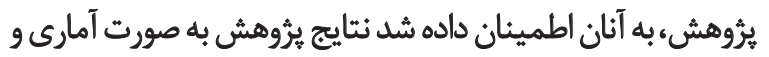

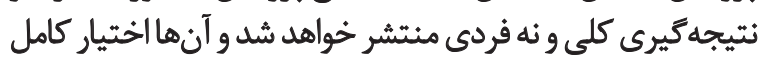

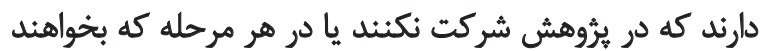

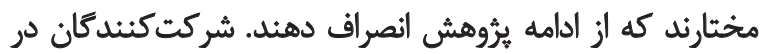

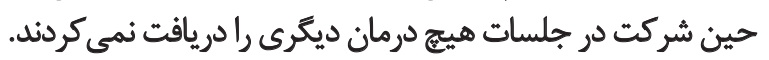

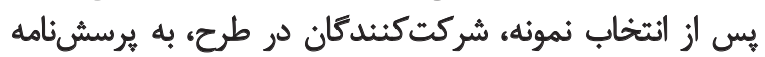

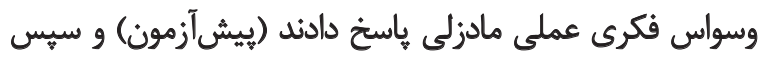

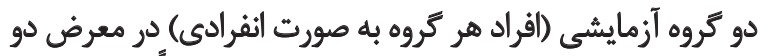

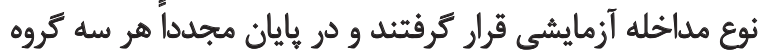

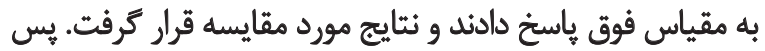

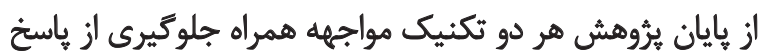

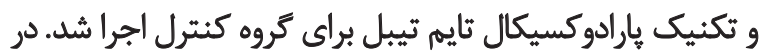

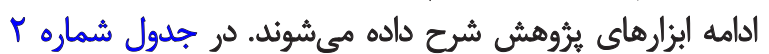

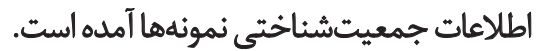

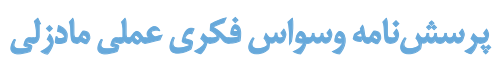

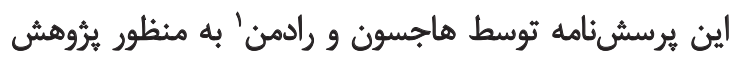

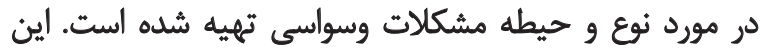

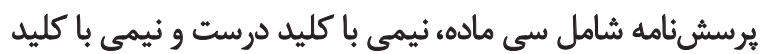

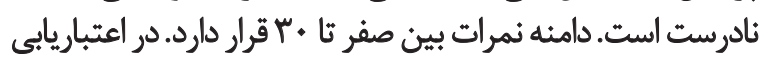

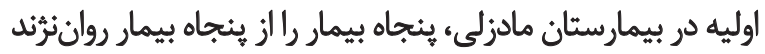

1. Hodgson and Radman 


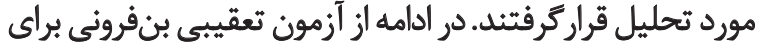

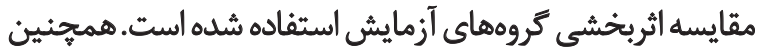

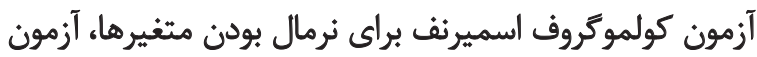

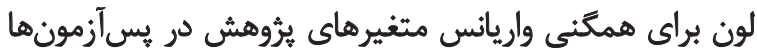

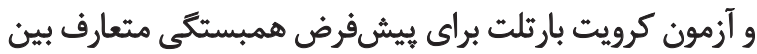

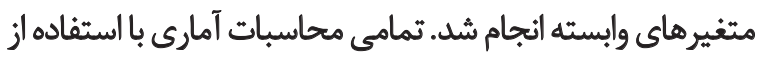
نسخه ل1 انرمافزار آمارى SPSS انجام كرفته است.

يافتهها

با توجه به نتايج جدول شماره با، در هارادوكسيكال ثنايم تيبل

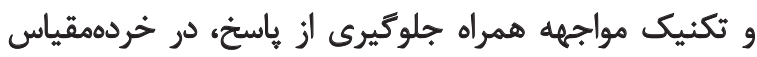

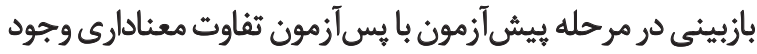

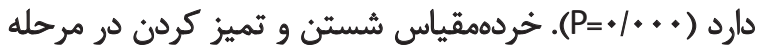

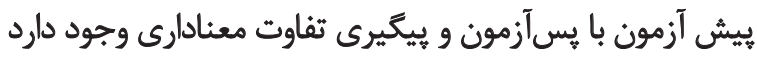

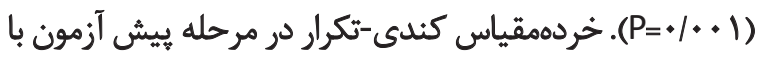

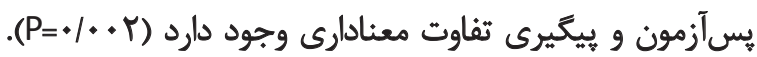

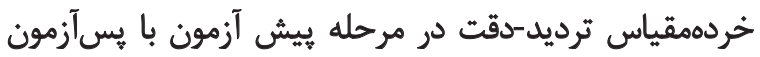

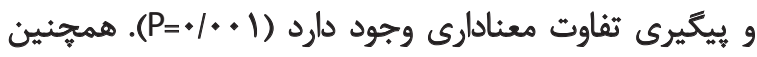

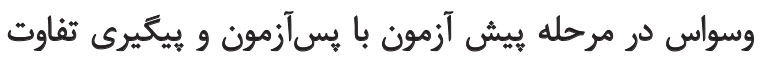

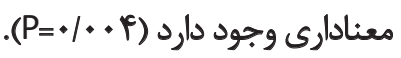

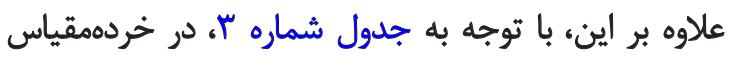

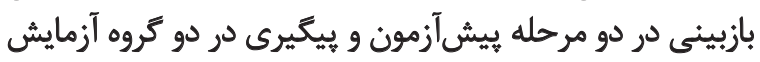

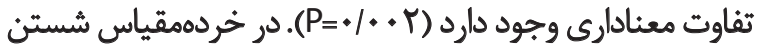

\section{تكنيك بارادوكسيكال تائم ثيبل}

كروه آزمايشى اين شيوه هم جهارجلسه هفتلكى نوددقيقه-

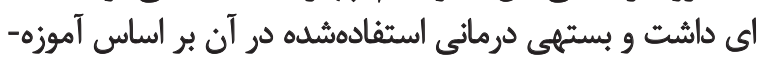

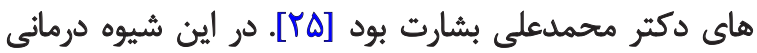

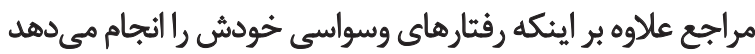

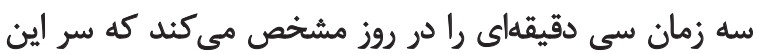

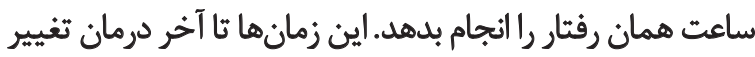

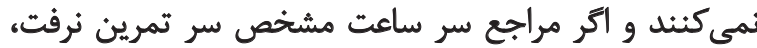

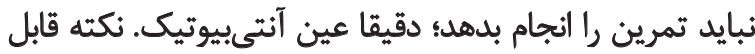

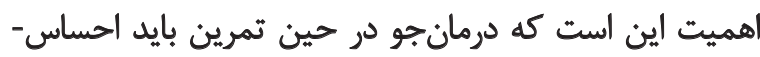

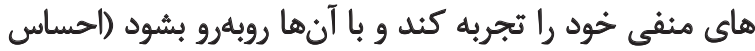

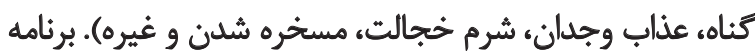

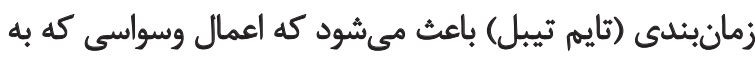

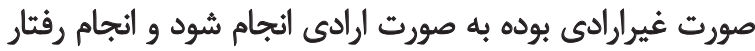

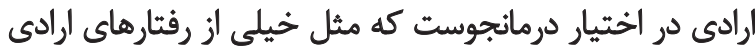

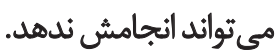
روش تحليل دادهها

در ئروهش حاضر هم از روشهاى آمارى توصيفى اعم از

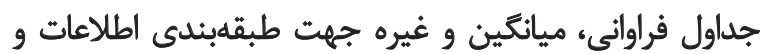

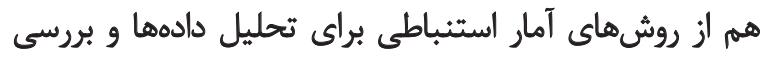

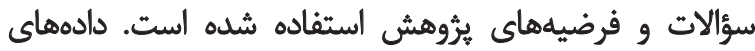
بيش آزمون و يس آزمون توسط روش آمارى تحليل كوواريانس

جدول r. ويزّكى هاى جمعيتشناختى نمونه

\begin{tabular}{|c|c|c|c|c|}
\hline \multicolumn{3}{|c|}{ تعداد (درصد) } & \multirow{2}{*}{ هتغيرها } & \\
\hline كروه كنترل & كروهدريافت كتنده تكنيك هيار ادوكسيكال تايم تيبل & كروه دريافت كنثده تكنيك مواجهه همراه جلوكيرى & & \\
\hline$\Delta(\Psi / M T)$ & $\wedge(\Psi / \Delta \Psi)$ & $9\left(\varepsilon_{0}\right)$ & دييلم & \\
\hline$\Lambda(\Psi / \Delta M)$ & $F(V / T E)$ & $f(Y e / N)$ & 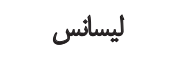 & 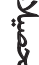 \\
\hline$r(r / M r)$ & $r\left(r_{0}\right)$ & $r(I r / \mu)$ & بالاتر از ليسانس & \\
\hline 1. (V/EF) & $\Pi(r / M)$ & $11(r / M r)$ & نj & \\
\hline$\Delta(N / M T)$ & $F(N / T E)$ & $F(N / T E)$ & هرد & \\
\hline$\wedge(H / \Delta H)$ & $\Lambda(r / \Delta r)$ & $\wedge\left(\Psi / \Delta H^{\Psi}\right)$ & خانهدار & \\
\hline$I(V / 8)$ & $r(\Psi / N)$ & $r(\Psi / / \Psi)$ & بيكار & $\bar{\xi}$ \\
\hline$\varepsilon\left(f^{e}\right)$ & $\Delta(\Psi / M T)$ & $\Delta(\Psi / M T)$ & شاغل & \\
\hline
\end{tabular}


جدول r. ميانكين و انحراف معيار نمرات آزمون وسواس (تعداد نموئه: هه)

\begin{tabular}{|c|c|c|c|c|}
\hline \multicolumn{3}{|c|}{ مياتكين 土 انحراف معيار } & \multirow{2}{*}{ كروهها } & \multirow{2}{*}{ مقياسها } \\
\hline يبئيرى & تيسآزمون & بيشئالزمون & & \\
\hline $4 / m \pm 1 / 11$ & $e / f \pm V / R f$ & $\Delta / f \pm 1 / f \Delta$ & تكنيك مواجهه همراه جلوكيرى از ياسخ & \\
\hline$f / Y \pm 1 / \circ q$ & $r / V \pm V / \mathcal{E}$ & $\Delta / Y \pm V / * \Lambda$ & تكنيك هارادوكسيكال تاييم تييل & 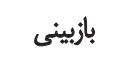 \\
\hline$\Delta / A \subset \pm . / 99$ & rarturr & $\Delta / M \pm I / \cdot \varphi$ & كروه كثترل & \\
\hline$\Delta / F \& \pm 1 / \Delta$ & $F / A F \pm 1 / F \Delta$ & $8 / 48 \pm 1 / 99$ & تكنيك مواجهه همراه جلوكيرى از ياسخ & \\
\hline$\Delta / Y C \pm 1 / \& \&$ & $f / F \pm V / * \Delta$ & $g / r \pm v / r q$ & تكنيك بارادوكسيكال ثايم ثييل & تميز كردن و \\
\hline$V / \cdot G \pm V / \cdot r$ & $g / r r \pm 1 / r q$ & $g / Y \pm V / 81$ & كروه كنترل & \\
\hline$r / / r \pm \| / / r$ & $r / . \& \pm V / . r$ & $r / q T \pm 1 / \pi r$ & تكنيك هواجهه همراه جلوكيرى از ياسخ & \\
\hline$r \% \pm \cdot / A P$ & $r / \& \& \pm 1 /+\varphi$ & $P / M T \pm \cdot M$ & تكنيك بارادوكسيكال ثايم تييل & كندي تكرار \\
\hline$r / q u \pm V / \cdot q$ & F/TED./M & $\Delta / \cdot \varphi \pm r / T \Delta$ & كروه كتترل & \\
\hline T/KE $\pm 1 / T V$ & $r / \mu+1 / 11$ & $r / I T \pm I / T$ & تكنيك مواجها همراه جلوكيرى از ياسخ & \\
\hline$r / q \Psi \pm \cdot / M$ & $r / F E \pm 1 / T$ & $r / \cdots \pm 1 / M^{\mu}$ & تكنيك يارادوكسيكال ثايم تيبل & ترديد دقت \\
\hline$r / M \pm \pm / M V$ & $r / N E \pm 1 / \Delta q$ & $1 / .8 \pm 1 / .9$ & كروه كثترل & \\
\hline $\mid F / T E \pm T M$ & IF/AYET/TQ & $\mid V / M T \pm r / \cdot 1$ & تكنيك هواجهه همراه جلوكيرى از هاسخ & \\
\hline $1 r / F \pm 1 / 9 \Delta$ & $|r / \cdot \pm \pm| / A \mid$ & IV/OrII/99 & تكنيك هارادوكسيكال ثنايم تييل & 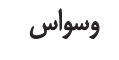 \\
\hline WrE \pm T/Fq & $W|r \pm T / F|$ & $|V / N E \pm T / F|$ & كروه كتترل & \\
\hline
\end{tabular}

انقانق

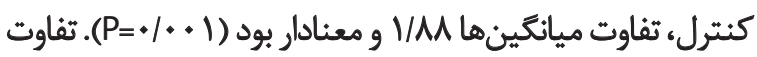

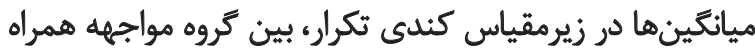

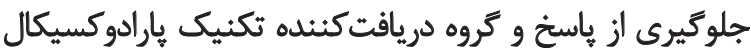

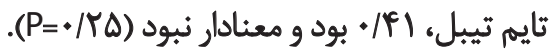

بين كروه مواجهه همراه جلوكيرى از ياسخ و كروه كنترل،

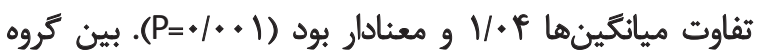

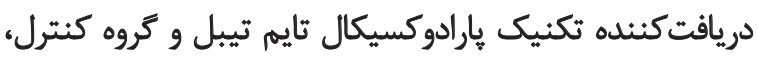

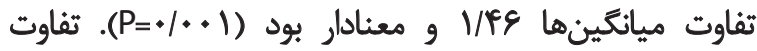

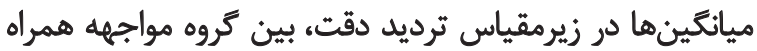

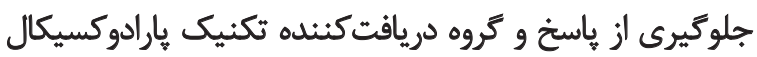

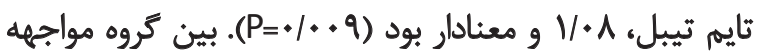

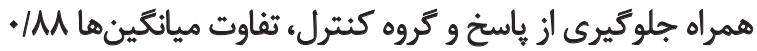

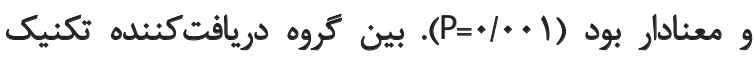

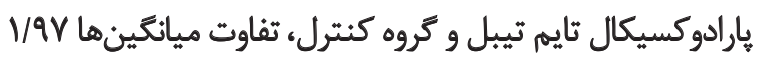

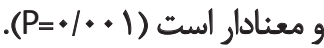

تفاوت ميانكينها در وسواس، بين كروه مواجهه همراه

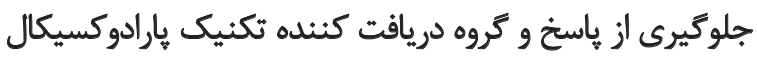

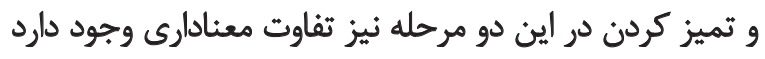

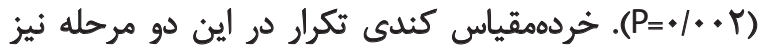

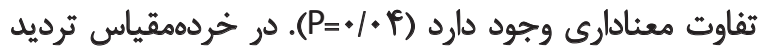

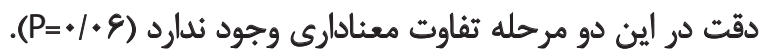

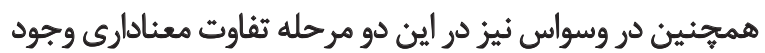

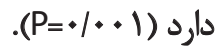

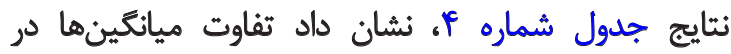

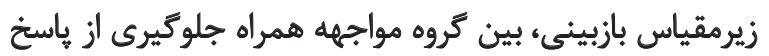

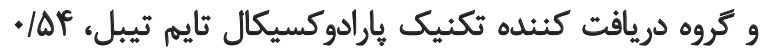

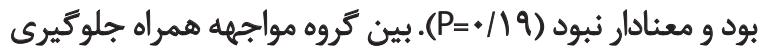

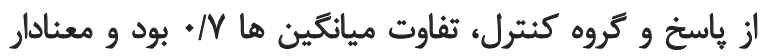

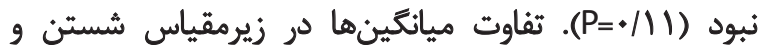

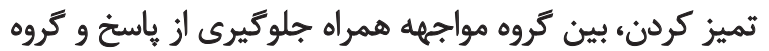

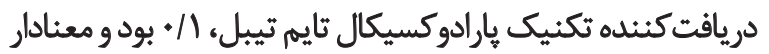

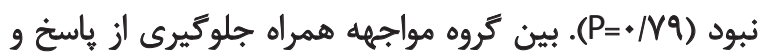

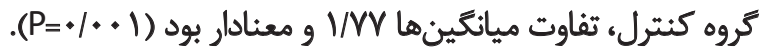

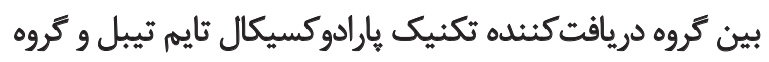




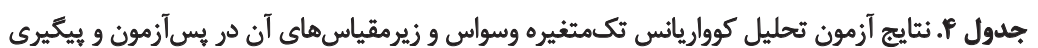

\begin{tabular}{|c|c|c|c|c|c|c|}
\hline سطح معنادارى & $F$ & مجذذورات & درجه آزادى & مجذورات & منابع تغيير & زمان اندازهكيرى \\
\hline .1 .1 & $F / T T$ & $\Delta / \Delta$ & r & $11 / . *$ & بازيينى & \\
\hline $.1 . .1$ & $11 / 4$ & $19 / \mathrm{rA}$ & $r$ & ra/or & شستن و تميز كردن & \\
\hline $.1 . .1$ & Nrs & $V / M r$ & r & $10 / 4 F$ & كندى تكرار & يس آزمون \\
\hline $.1 .+1$ & $1 r / \cdot r$ & $1 r / 199$ & $r$ & ry/va & ترديد دقت & \\
\hline .1 .01 & $\Delta T / \backslash \Delta$ & $111 / \mathrm{r}$ & $r$ & $r T r / M P$ & وسواس & \\
\hline$\% r$ & $V / V$. & N/T & r & IEIFA & بازيينى & \\
\hline.$/ . r$ & $V / F q$ & Ir/IF & r & re/rq & شستن و تميز كردن & \\
\hline .1 .4 & $r / \Delta$ & $r / v G$ & r & $V / \Delta Y$ & كندى تكرار & ييكيرى \\
\hline .1 .8 & $r / q$ & $r / *$ & $r$ & $91 .$. & ترديد دقت & \\
\hline $.1 .+1$ & $r M / T I$ & VNTE & r & lQVVMr & وسواس & \\
\hline
\end{tabular}

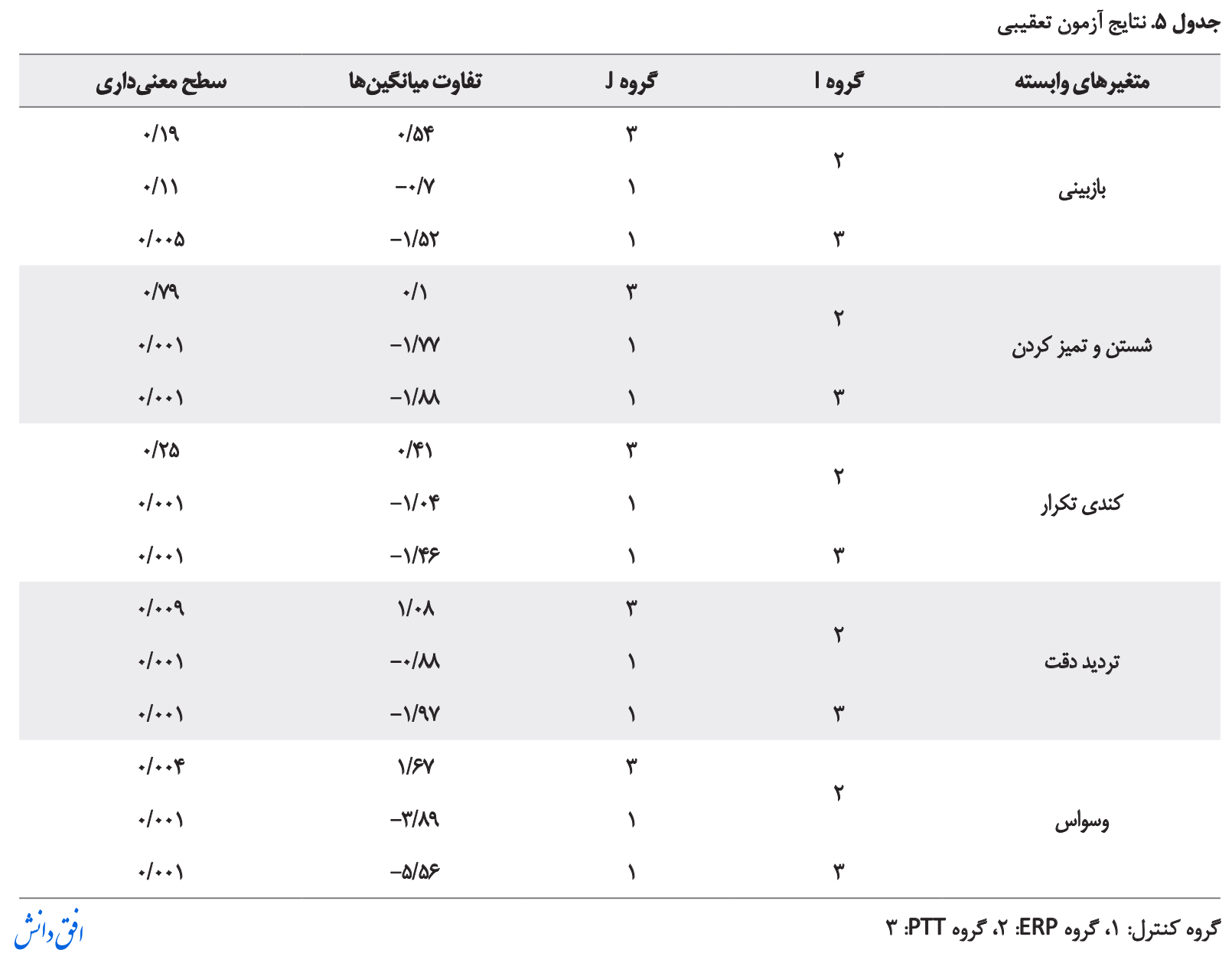


طريقى است كه مانع ايجاد مقاومت ويا تحليل ادراكى بيجيديده

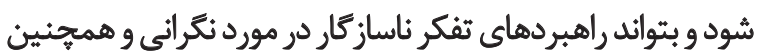

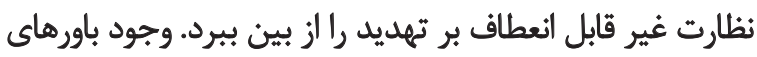

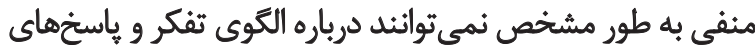

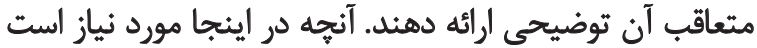

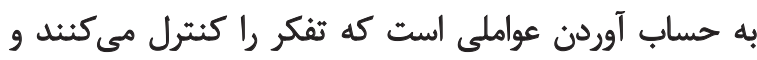

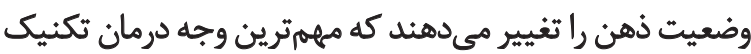
يارادوكسيكال تايم تيبل است.

يكى از دلايل حاصل شدن اين نتيجه كه تكنيك مواجهيه

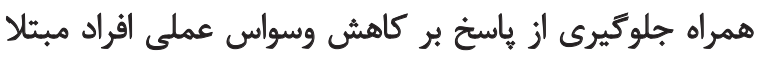

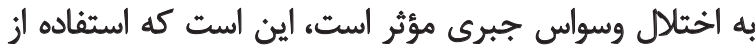

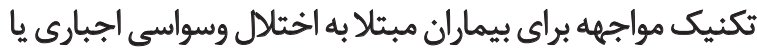

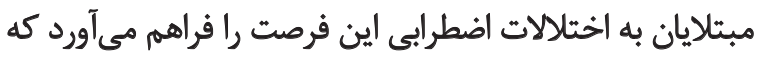

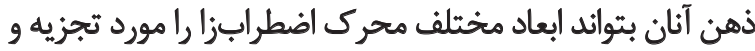

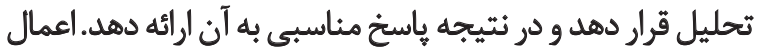

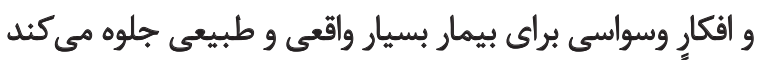

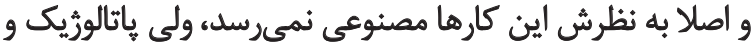
آزاردهينده أست.

همجنين عمل و فكر وسواسى دستورى نيست، يعنى در

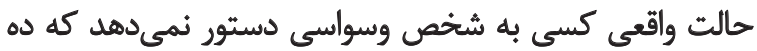

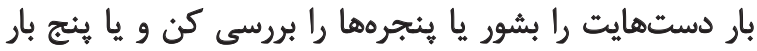

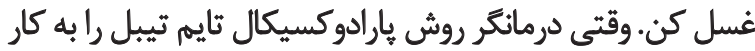

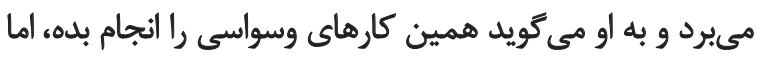

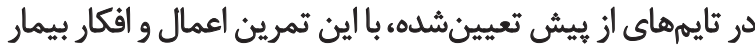

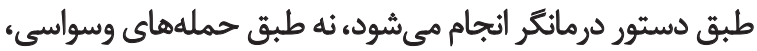

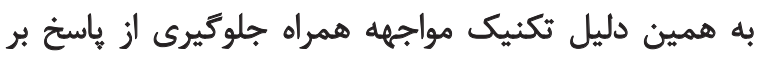

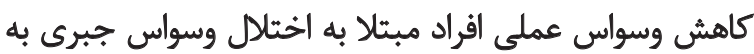

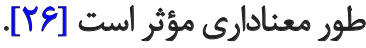

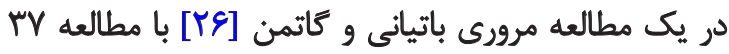

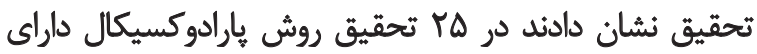

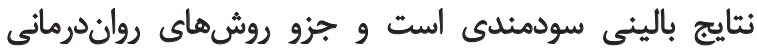

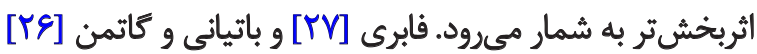

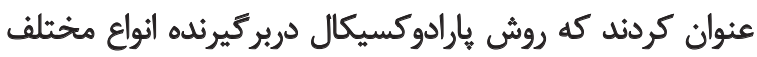

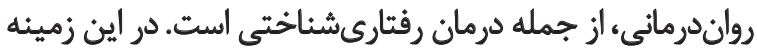

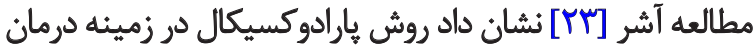

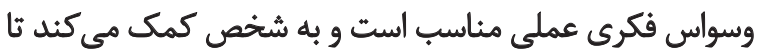

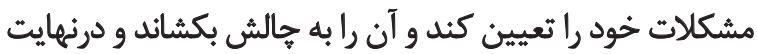

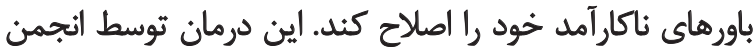

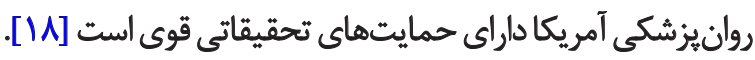

تنيجنَيرى

درنهايت با در نظر كرفتن اين مسئله كه ميانكين نمرات

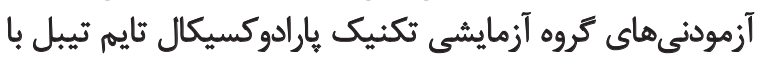

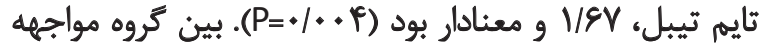

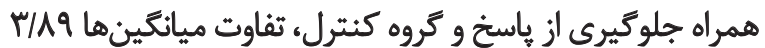

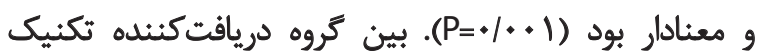

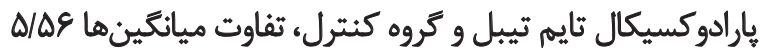

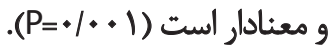

جدول شماره ه، ميانكين و انحراف معيار نمرات آزمودنى هاى

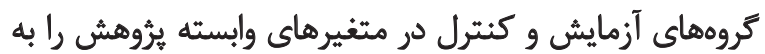

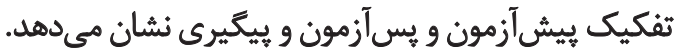

ث

هدف اين ثروهش مقايسه اثربخشى تكنيك مواجها همريك

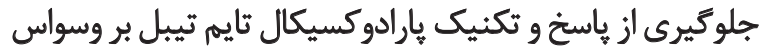

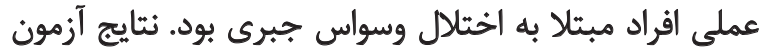

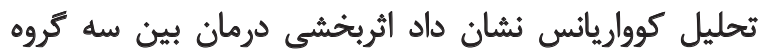

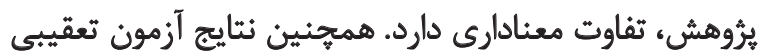

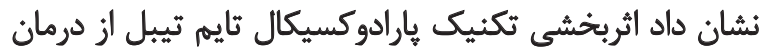

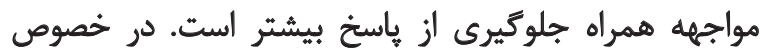

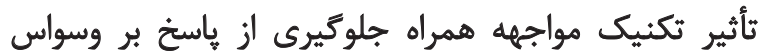

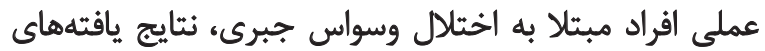

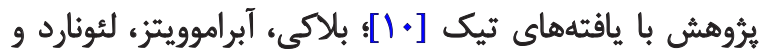

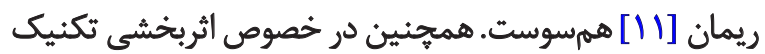

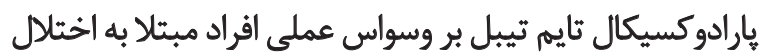

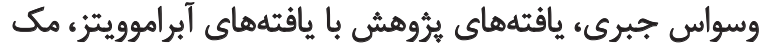

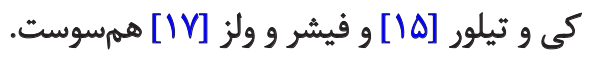

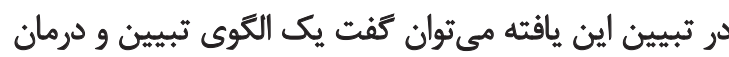

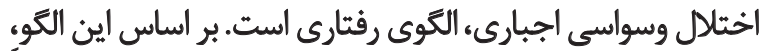

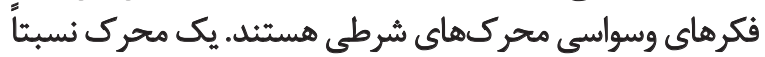

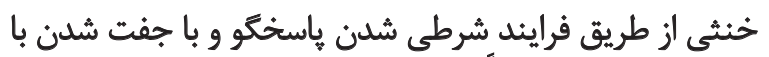

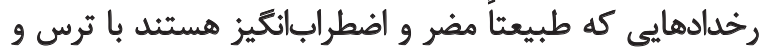

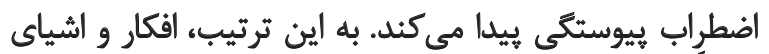

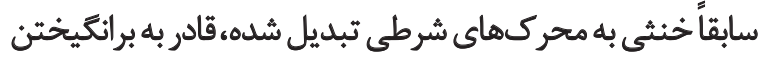

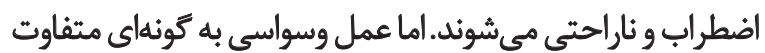

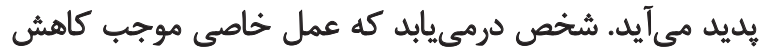

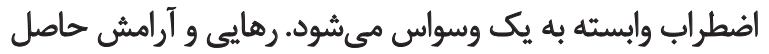

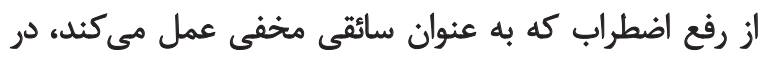

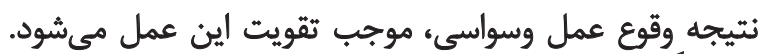

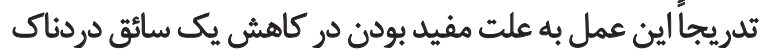

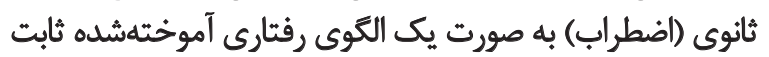

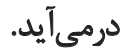

در تبيين اثربخشى الكوى ثارادوكسيكال تايم تيبل در يرؤرهش

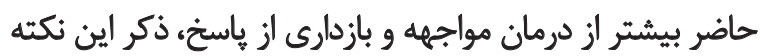

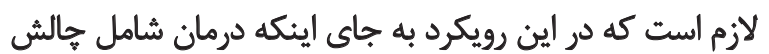
با افكار مزاحم و باورهاى ناكارآمد باشد، شامل ائك ارتباط دان با افكار به 


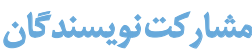

جمعآورى داده: صديقه احمدى؛ ايده اصلى: آذرميدخت

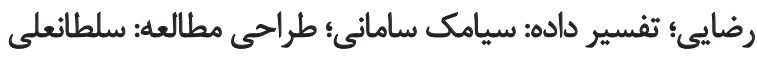

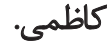

$$
\text { تعارض منأع }
$$

هيجگونه تعارض منافعى توسط نويسندكان مقاله بيان نشده
كروه آزمايشى درمان مواجهه و بازدارى از ياسخ و همجنين كروه

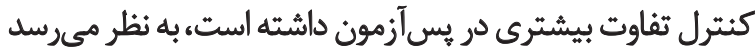

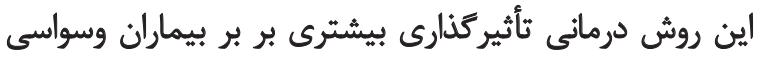

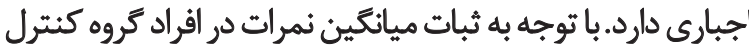

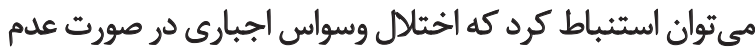

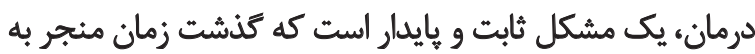

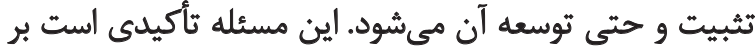

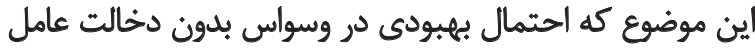

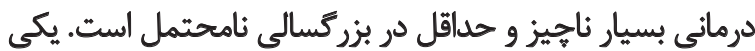

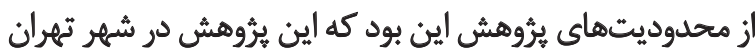

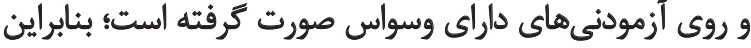
تعميم آن به ساير جوامع بايد با احتياط صورت كيرد.

در اين بثوهش بسيارى از عوامل روانشناختى تأثير كذار كنترل

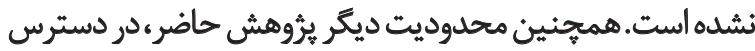

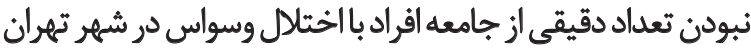

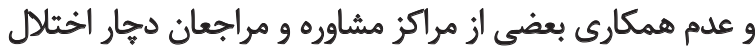

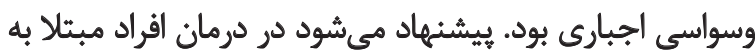

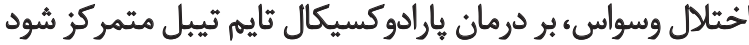

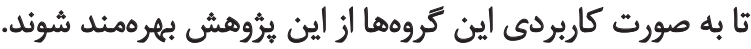

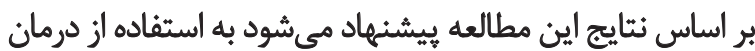

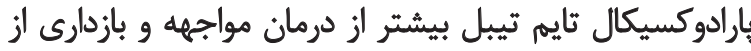

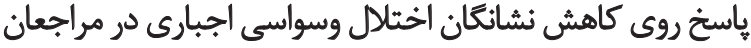

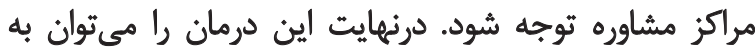

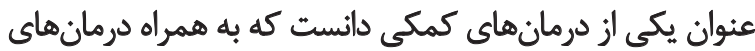

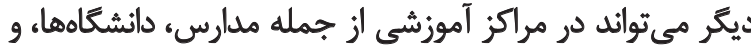

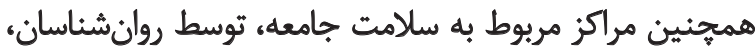

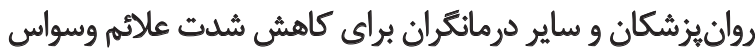

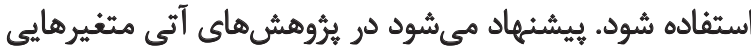

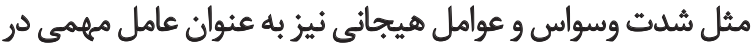

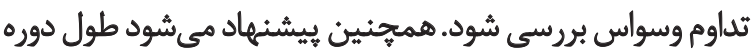

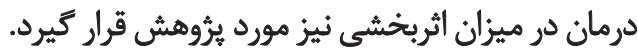

مالاحظات اخلاقى

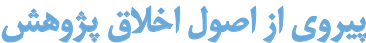

از مشاركت كنندكان در مطالعه رضايت كتبي و آكاهانه اخذ

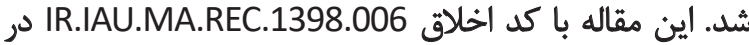
دانشكاه آزاد اسلامى واحد مرودشت به ثبت اخلاق رسيده است.

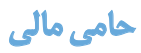

اين مقاله بركرفته از رساله دكتراى صديقه احمدى در دانشعًاه

$$
\text { آزاد اسلامى واحد مرودشت است. }
$$




\section{References}

[1] Vikas A, Avasthi A, Sharan P. Psychosocial impact of obsessivecompulsive disorder on patients and their caregivers: A comparative study with depressive disorder. International Journal of Social Psychiatry. 2011; 57(1):45-56. [DOI:10.1177/0020764009347333]

[2] Kessler RC, Chiu WT, Demler O, Walters EE. Prevalence, severity, and comorbidity of 12-month DSM-IV disorders in the National Comorbidity Survey Replication. Archives of General Psychiatry. 2005; 62(6):617-27. [DOI:10.1001/archpsyc.62.6.617] [PMID]

[3] Ruscio AM, Stein DJ, Chiu WT, Kessler RC. The epidemiology of obsessive-compulsive disorder in the National Comorbidity Survey Replication. Molecular Psychiatry. 2010; 15(1):53. [DOI:10.1038/ mp.2008.94] [PMID] [PMCID]

[4] Amani Malahat, Abolqasemi Abbas, Ahadi Batul, Narimani, Mohammad. [The prevalence of obsessive-compulsive disorder among women aged 20-40 in Ardabil city (Persian)]. Journal of Basic Principles of Mental Health. 2013; 15(59):233-44. http://eprints.mums. ac.ir/4452/

[5] Safarulzadeh F, Hashemlo L, Bagheri F, Motaarefi H. [The Prevalence of Obsessive-Compulsive Disorder in Khoy University Students in 1391 (Persian)]. Journal of Psychiatric Nursing. 2014; 2(2):13-21. http://ijpn.ir/article-1-329-en.html

[6] Salehi S, Mousavi SA M, Sarichlou ME, Shahid Beheshti SH. [Investigation of demographic characteristics and symptoms of obsessivecompulsive disorder (Persian)]. Journal of Sabzevar University of Medical Sciences. 2014; 21 (2):343-351. https://www.sid.ir/en/Journal/ViewPaper.aspx?ID=398230

[7] O'Donohue WT, Fisher JE, editors. General principles and empirically supported techniques of cognitive behavior therapy. United States: John Wiley \& Sons; 2009.

[8] American Psychiatric Association. Diagnostic and statistical manual of mental disorders (DSM- ${ }^{\circledR}$ ). Philadelphia: American Psychiatric Association; 2013. [DOI:10.1176/appi.books.9780890425596]

[9] Wells A. Cognitive therapy of anxiety disorders: A practice manual and conceptual guide. United States: John Wiley \& Sons; 2013.

[10] Tice AM. Adapting an exposure and response prevention manual to treat youth obsessive-compulsive disorder and comorbid anxiety disorder: The case of" Daniel". Pragmatic Case Studies in Psychotherapy. 2019; 15(1):1-74. [DOI:10.14713/pcsp.v15i1.2043]

[11] Blakey SM, Abramowitz JS, Leonard RC, Riemann BC. Does exposure and response prevention behaviorally activate patients with obsessive-compulsive disorder? A Preliminary Test. Behavior therapy. 2019; 50(1):214-24. [DOI:10.1016/j.beth.2018.05.007] [PMID]

[12] Leahy RL, Holland SJ, McGinn LK. Treatment plans and interventions for depression and anxiety disorders. United States: Guilford Press; 2011.

[13] Foa EB, Liebowitz MR, Kozak MJ, Davies S, Campeas R, Franklin ME, et al. Randomized, placebo-controlled trial of exposure and ritual prevention, clomipramine, and their combination in the treatment of obsessive-compulsive disorder. American Journal of Psychiatry. 2005; 162(1):151-61.[PMID]

[14] Laforest M, Bouchard S, Bossé J, Mesly O. Effectiveness of in virtuo exposure and response prevention treatment using cognitive-behavioral therapy for obsessive-compulsive disorder: A Study based on a single-case study protocol. Frontiers in Psychiatry. 2016; 7:99. [DOI:10.3389/fpsyt.2016.00099] [PMID] [PMCID]
[15] Abramowitz JS, McKay D, Taylor S, editors. Clinical handbook of obsessive-compulsive disorder and related problems. United States: JHU Press; 2008.

[16] Franklin V. Man's Search for Meaning [N. Salehian, M. Milani tran]. Tehran: Dorsa Publications; 2011.

[17] Fisher PL, Wells A. How effective are cognitive and behavioral treatments for obsessive-compulsive disorder? A clinical significance analysis. Behaviour Research and Therapy. 2005; 43(12):1543-58. [DOI:10.1016/j.brat.2004.11.007] [PMID]

[18] Lewis MH. Amelioration of obsessive-compulsive disorder using paradoxical intention. InLogotherapy and Existential Analysis (pp. 175-178). Boston: Springer; 2016. [DOI:10.1007/978-3-31929424-7 16]

[19] Graber AV. Viktor Frankl's logotherapy: Meaning-centered counseling. United Kingdom: Wyndham Hall; 2004.

[20] Stiefelmann H. Paradoxical intention: A homeopathic psychotherapeutic approach. Revista de Homeopatia. 2015; 78(1/2):9-20.

[21] Ascher LM, Efran JS. Use of paradoxical intention in a behaviora program for sleep onset insomnia. Journal of Consulting and Clinical Psychology. 1978; 46(3):547. [DOI:10.1037/0022-006X.46.3.547]

[22] Ameli M, Dattilio FM. Enhancing cognitive behavior therapy with logotherapy: Techniques for clinical practice. Psychotherapy. 2013 50(3):387. [DOI:10.1037/a0033394] [PMID]

[23] Ascher LM. Paradoxical intention and related techniques. In Encyclopedia of Cognitive Behavior Therapy 2005 (pp. 264-268). Boston: Springer; 2005. [DOI:10.1007/0-306-48581-8_74]

[24] Delavar A. [Research methods of psychology and education (Persian)]. Tehran: Virayesh; 2017.

[25] Besharat M. [Assessing the effectiveness of paradox therapy on obsessive-compulsive disorder: A case study (Persian)]. Psychological Studies. 2017; 14(4):7-36. [Doi:10.22051/psy.2019.23631.1801]

[26] Batthyany A, Guttmann D. Empirical research in logotherapy and meaning-oriented psychotherapy: An annotated bibliography. United States: Zeig, Tucker \& Theisen; 2006

[27] Fabry D D S. (2010). Evidence base for paradoxical intention: Reviewing clinical outcome studies. International Forum for Logotherapy, 33(1), 21-9. https://psycnet.apa.org/record/2010-16141-005 